\title{
CELL ADDRESSING AND TRAPPING USING NOVEL OPTOELECTRONIC TWEEZERS
}

\author{
Pei Yu Chiou' ${ }^{1}$, Wilson Wong ${ }^{2}$, James C. Liao' ${ }^{2}$, Ming C. Wu ${ }^{1}$ \\ ${ }^{\text {l}}$ Department of Electrical Engineering, ${ }^{2}$ Department of Chemical Engineering, \\ University of California at Los Angeles (UCLA), CA 90095, USA \\ TEL: (310) 825-7338, FAX: (310) 794-5513, Email: peiyu@icsl.ucla.edu
}

\begin{abstract}
We present novel optoelectronic tweezers (OET) that are capable of trapping and transporting multiple biological cells. This is based on a new mechanism called light induced dielectrophoresis proposed by our group [1]. OET allows a focused optical beam to create a virtual electrode on a photoconductive surface, producing a highly non-uniform electric field. This enables optical addressing of dielectrophoresis forces with a spatial resolution of $\sim 1 \mu \mathrm{m}$. The optical intensity required is three orders of magnitudes lower than that of conventional optical tweezers. By scanning the laser beam, the trapped cells can be moved to any position on a 2D surface. Experimentally, we have successfully demonstrated the concentration and transport of multiple $E$. coli cells using a HeNe laser with a focused spot size of $17 \mu \mathrm{m}$. Optical power as low as $8 \mu \mathrm{W}$ is sufficient trap the $E$ coli cells.
\end{abstract}

\section{INTRODUCTION}

Optical tweezers have been widely used to trap, observe, and manipulate biological cells since they were first demonstrated by Ashkin [2-4]. Optical tweezers rely on optical gradient force, which competes with radiation pressure. Therefore, very tight focusing is needed to produce a stable optical trap. The trapping area is much smaller than typical cells and as a result, the force exerted on the cells is very non-uniform. In addition, optical tweezers require very high optical power (1 to $100 \mathrm{~mW}$ ) to produce sufficient trapping force $(\sim 1$ to $100 \mathrm{pN})$. The high optical power density could result in photodamage of cells [3], though the use of infrared lasers relieves the "opticution" problem [4].

Instead trapping cells and particles directly using optical forces, optical control of electrokinetic forces has been proposed recently. Ozkan et al. proposed light induced electrophoresis, which allows optical addressing of charged polymer beads on semiconductor surface [5]. Our group proposed the use of light induced dielectrophoresis to address electrically neutral or charged particles optically [1]. We called this mechanism optoelectronic tweezers (OET)

The principle of OET is illustrated in Fig. 1. The optical energy is first converted to electrical energy by a photoconductor, which in turn controls the dielectrophoresis (DEP) force to interact with cells. The OET offers several advantages for manipulating cells: first, the optical power can be drastically reduced because of the "optoelectronic gain" in photoconductors. We have shown that optical power as low as $1 \mu \mathrm{W}$ is sufficient to move a $25 \mu \mathrm{m}$ latex bead at a speed of $4.5 \mu \mathrm{m} / \mathrm{s}$. Second, tight optical focusing is not required. The trapping area can be tailored to match the cell size. Third, larger forces (several hundred pico Newtons) can be produced by OET.

In this paper, we demonstrate for the first time the trapping and moving of live $E$. Coli cells using optoelectronic tweezers. Cell moving speed of $120 \mu \mathrm{m} / \mathrm{s}$ has been observed.

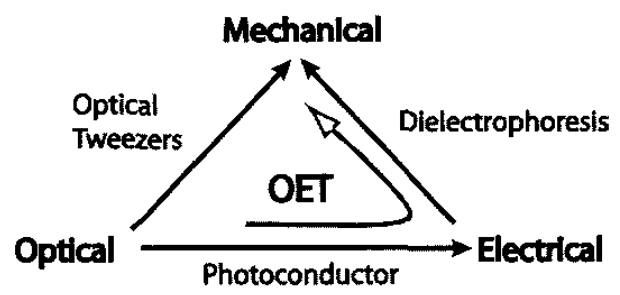

Fig.1 Energy conversion of optoelectronic tweezers (OET).

\section{PRINCIPLE OF OPTOELECTRONIC TWEEZERS}

Optoelectronic tweezers trap cells using light induced dielectrophoresis. Dielectrophoresis (DEP) has been widely used in manipulating cells and micro-particles [6]. It utilizes the interaction force between a non-uniform electric field and the induced dipole of the particle. The DEP force can be expressed analytically by

$$
\begin{aligned}
& F_{d e p}=2 \pi a^{3} \varepsilon_{m} \operatorname{Re}\left[K^{*}(\omega)\right] \nabla\left(E^{2}\right) \\
& K^{*}(\omega)=\frac{\varepsilon_{p}^{*}-\varepsilon_{m}^{*}}{\varepsilon_{p}^{*}-2 \varepsilon_{m}^{*}}, \varepsilon_{p}^{*}=\varepsilon_{p}-j \frac{\sigma_{p}}{\omega}, \varepsilon_{m}^{*}=\varepsilon_{m}-j \frac{\sigma_{m}}{\omega}
\end{aligned}
$$

where $E$ is the electric field strength, $a$ is the particle radius, $\varepsilon_{m}$ and $\varepsilon_{p}$ are the permittivities of the surrounding medium and the particle, respectively, $\sigma_{m}$ and $\sigma_{p}$ are the conductivities of the medium and the particle, respectively, $\omega$ is the angular frequency of the applied electric field. $K^{*}(\omega)$ is the Clausius-Mositti factor. The real part of $K^{*}(\omega)$, or $\operatorname{Re}\left[K^{*}(\omega)\right]$, has a value between 1 and $-1 / 2$, depending on the polarizabilities of the medium and the particle and on the frequency of the applied ac electric bias. If $\operatorname{Re}\left[K^{*}(\omega)\right]>0$, the 
particle will move towards higher electric field region and this is called positive DEP. On the other hand, if $\operatorname{Re}\left[K^{*}(\omega)\right]$ $<0$, the particle will move in the opposite direction and this is called negative DEP. In this paper, we demonstrate the trapping of $E$. Coli cells using light induced positive DEP force. Since DEP force is proportional to the volume of the particle and the gradient of $E^{2}$, a highly non-uniform field is required to transport cells with sizes of $1 \sim 2 \mu \mathrm{m}$, such as $E$. Coli.

Figure 2 shows the schematic structure of the OET. The liquid containing the cells is sandwiched between a photosensitive surface (top) and a transparent conductive surface (bottom) made of indium-tin oxide (ITO). The top surface is fabricated on an ITO glass substrate and is comprised of three pattern-less layers: a 5-nm-thick aluminum layer, a 1- $\mu$ m-think amorphous silicon layer (photoconductor), and a 20 -nm-thick silicon nitride layer. The top wafer is flipped over and placed on top of the bottom wafer with a $15-\mu \mathrm{m}$-thick photoresist spacer. The top and the bottom surfaces are not physically bonded together. Instead, since both surfaces are hydrophilic, they are pulled towards each other by the surface tension of the liquid until stopped by the photoresist spacer. An ac bias is applied between the top and the bottom ITO electrodes.

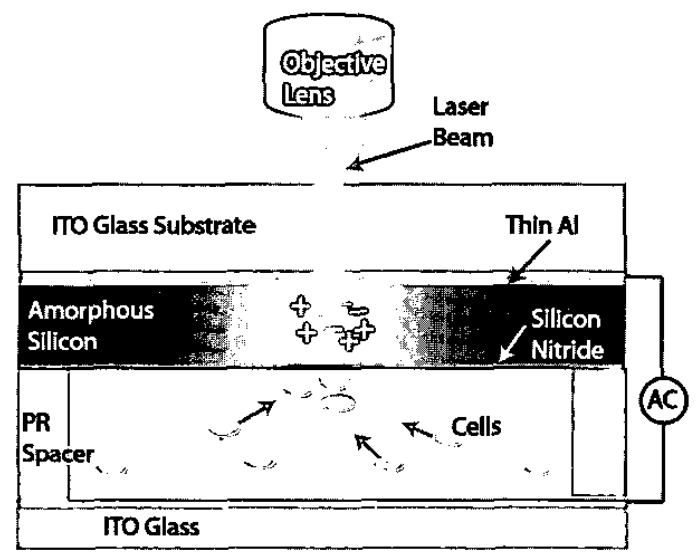

Fig.2 Schematic structure of optoelectronic tweezers. A virtual electrode is created at the laser illuminated area.

Intrinsic amorphous silicon is a photoconductor with a dark conductivity of $10^{-8} \mathrm{~S} / \mathrm{cm}$ to $10^{-10} \mathrm{~S} / \mathrm{cm}$. It behaves like an insulator in the dark. When a laser beam is focused on the photoconductor, the photogenerated carriers cause the local conductivity to increase by several orders of magnitudes. This allows the laser beam to create a localized electrode on the photoconductor and generate a non-uniform electric field in the liquid layer. The resolution of the light-patterned electrode is ultimately limited by the focused spot size and the ambipolar diffusion length in photoconductor. The ambipolar diffusion length in amorphous $\mathrm{Si}$ is as small as 115 $\mathrm{nm}[7]$.

\section{SIMULATION OF ELECTRIC FIELD DISTRIBUTION}

Figure 3 shows the simulated electric field distribution in the liquid layer for a $17 \mu \mathrm{m}$ spot generated by a focused laser beam and a bias voltage of $10 \mathrm{~V}$. The conductivity of the liquid is $1 \mathrm{mS} / \mathrm{m}$. The photoconductivity of the amorphous silicon layer is assumed to have a Gaussian distribution, following the profile of the incident light, with a peak conductivity of $10 \mathrm{mS} / \mathrm{m}$ at the center. The three-dimensional electrical field distribution is calculated using FEMLAB.

The electric field strengths at three different heights above the photosensitive surface are plotted in Fig. 4. Since the DEP force is proportional to the gradient of $E^{2}$, the electric field distribution shows that the OET can generate strong DEP force within a radius of $\sim 20 \mu \mathrm{m}$ in the lateral direction. There is also a vertical gradient that will attract micro-particles towards the photoconductor surface. Both the lateral and the vertical gradients are strongest near the edge of the laser spot, similar to those generated by a physical electrode.

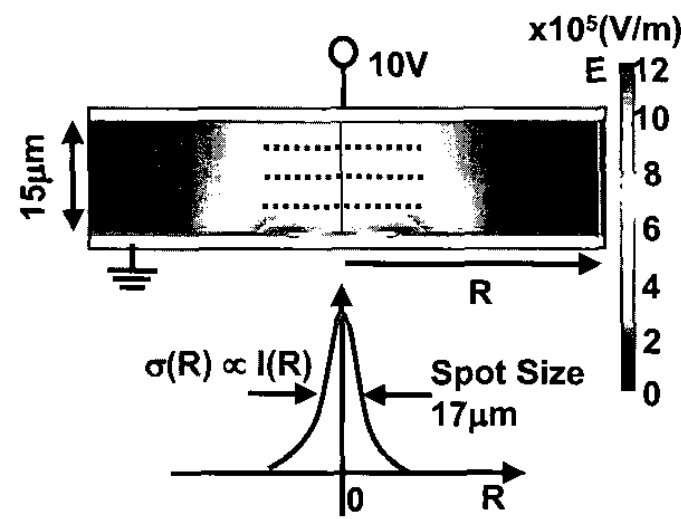

Fig.3 Electric field distribution in the liquid layer when the photoconductor is illuminated by a focused laser with $17 \mu \mathrm{m}$ spot size.

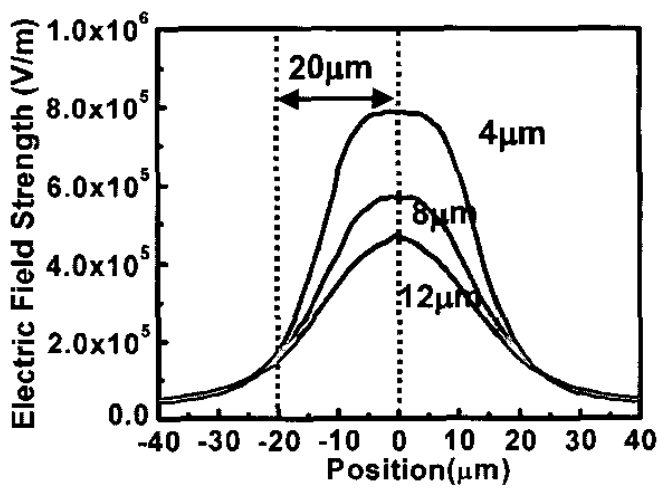

Fig.4 The electric field strength at three different heights above the photoconductive layer. 


\section{EXPERIMENT SETUP}

The experimental setup for trapping biological cells is shown in Fig. 5. The device is placed on an inverted microscope (Nikon TE2000E) with photosensitive surface side up. A $0.8 \mathrm{~mW}$ HeNe laser (wavelength $=632 \mathrm{~nm}$ ) is used to power the optoelectronic tweezers. A neutral density filter is added to control the optical power at the OET. The optical beam is delivered to the device through a $40 \times$ objective lens with a numerical aperture (NA) of 0.5 . The resulting focused spot size is $17 \mu \mathrm{m}$. The fluorescent image of the cells is captured through the bottom objective lens by a CCD camera.

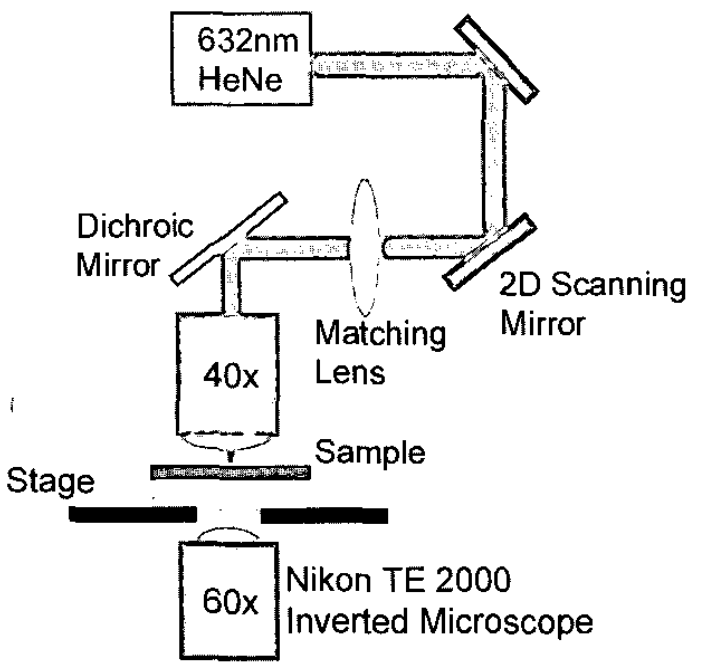

Fig.5 Experimental setup for trapping E. coli cells with optoelectronic tweezers

\section{RESULTS AND DISCUSSIONS}

When the laser beam is focused on a fixed spot, the OET attracts cells within the trapping area towards the center of the focus. It functions as a cell concentrator. Figure 6 shows the captured video images of the $E$. coli cells before and after the OET is turned on. In the experiment, we use the $E$. coli cells that can express green fluorescent protein (GFP) for the convenience of observation under fluorescent microscope. The liquid has a conductivity of $1 \mathrm{mS} / \mathrm{m}$. We apply a $100 \mathrm{kHz}$, 10 volts peak-to-peak ac electric bias between the top and the bottom ITO electrodes. The $E$. coli cells experience positive DEP force under these conditions. Before the laser is turned on, the electric field in the liquid is very weak and the $E$. coli cells are randomly distributed (Fig. 6(b)). When the laser is turned on, the cells within a $20 \mu \mathrm{m}$ radius start to move towards the laser beam, and eventually are trapped at the focal spot, as shown in Fig. 6(c). Due to the electric field gradient in the vertical direction, the cells are trapped right on top of the photosensitive surface.
When the laser beam is turned off, these trapped $E$. coli cells swim away. No photodamage is observed even for light in the visible wavelength. We have also investigated the minimum optical power required to operate this OET. Cell concentration is observed for optical power as low as $8 \mu \mathrm{W}$. The optical power level is three orders of magnitudes lower than that of conventional optical tweezers. The optical power density is more than five orders of magnitudes lower.

The concentrated cells can be moved to any arbitrary location by scanning the laser beam. Figure 7 shows the transport of multiple $E$. coli cells using a single scanning laser beam.

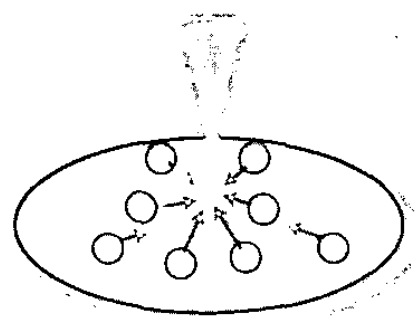

(a)

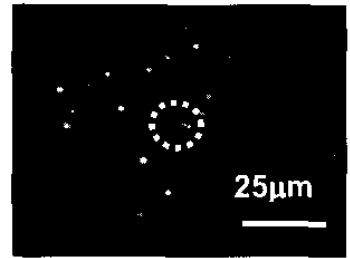

(b)

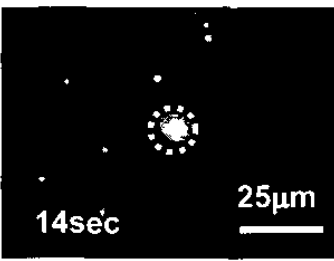

(c)
Fig.6 (a) Schematic diagram of cell concentrator. (b) Images of fluorescent E. coli cells before OET is turn on. (c) The same image after the OET is turned on for 14 seconds. The E. coli cells are "focused" by the OET to the laser spot.

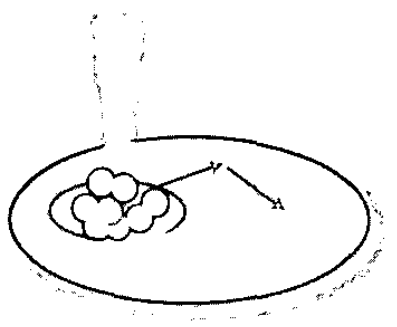

(a)

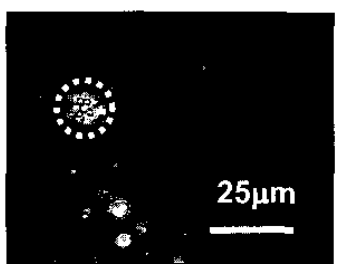

(b)

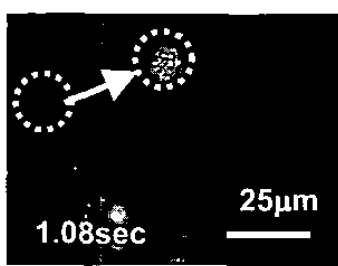

(c)
Fig. 7 Transport of multiple E. coli cells trapped by the OET. The E. coli cells remain trapped during the transport. 
To study the effective trapping area and the velocity of the cells being attracted to the traps, we recorded the trapping action and analyzed the video images frame by frame. The recording microscope is focused on the surface of the photoconductor to capture the trapped cells. We have measured the velocities of the trapped cells at $8,120,400$, and $800 \mu \mathrm{W}$. The OET traps work at all power levels. Figure 8 shows the measured velocities versus the radial distance from the center of the trap. At $800 \mu \mathrm{W}$, cells as far as $30 \mu \mathrm{m}$ away are attracted by the OET. Initially, they move at a relatively low speed of $5 \mu \mathrm{m} / \mathrm{s}$. The speed increases sharply when they are within $20 \mu \mathrm{m}$, eventually reaching a speed of $120 \mu \mathrm{m} / \mathrm{s}$ at about $15 \mu \mathrm{m}$ from the focal point. The transport speed becomes smaller after the peak value. The cells are stopped at $9 \mu \mathrm{m}$ from the center by the trapped cell cluster. This result matches very well with the simulated electric field distribution in Fig. 4. The maximum slope of the top curve ( $4 \mu \mathrm{m}$ above photoconductor) happens at about $15 \mu \mathrm{m}$ from the center.

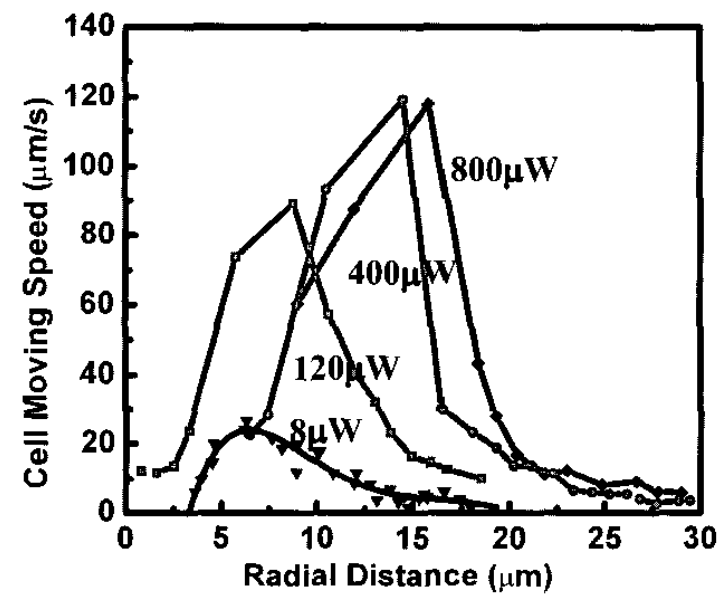

Fig.8 The moving speed of the E. Coli cells toward the center of the focused light spot. $R$ is the radial position of the cell.

The cell velocity is a function of the optical power. The peak velocity increases from $26 \mu \mathrm{m} / \mathrm{s}$ at $8 \mu \mathrm{W}$ to about 90 $\mu \mathrm{m} / \mathrm{s}$ at $120 \mu \mathrm{W}$. Above $120 \mu \mathrm{W}$, the peak velocity increases more slowly, and eventually saturate at about $200 \mu \mathrm{W}$. This can be explained by the following: when the optical power is lower than $120 \mu \mathrm{W}$, the photoconductor is not fully turned on, i.e., the conductivity is lower than but not negligible compared to the liquid. At about $200 \mu \mathrm{W}$, the conductivity of liquid becomes dominant in the electrical circuit, and most of the electric field drops across the liquid layer. Further increase of optical power does not change the peak electric field. However, the electric field distribution becomes more "square" like because of this saturation effect. The capturing area increases slightly after the peak field saturates.

It should also be pointed out that the minimum optical power required for OET depends on the liquid conductivity. The liquid conductivity used in current experiments is $1 \mathrm{mS} / \mathrm{m}$. The current laser power can actuate cells in liquid with conductivities up to $100 \mathrm{mS} / \mathrm{m}$. The optical power can be further reduced by shrinking the optical spot size. The current power level can be reduced by 100 times by decreasing the spot size from $17 \mu \mathrm{m}$ to $1.7 \mu \mathrm{m}$.

\section{CONCLUSIONS}

We have demonstrated for the first time the manipulation of biological cells using optoelectronic tweezers (OET). The optical power of OET is three orders of magnitudes lower than that of optical tweezers with comparable trapping force. It does not require tight optical focusing. Using a focused spot size of $17 \mu \mathrm{m}$, we have successfully demonstrated focusing of live $E$. coli cells with optical power as low as $8 \mu \mathrm{W}$. The optical power can be further reduced by shrinking the optical spot size. The low optical power and the large trapping area make OET an ideal candidate for manipulating cells. The low optical power also makes it possible to operate a large number of traps simultaneously using a spatial light modulator.

\section{ACKNOWLEDGEMENT}

This project is supported by Center for Cell Mimetic Space Exploration (CMISE), a NASA University Research, Engineering and Technology Institute (URETI), under award number \#NCC 2-1364.

\section{REFERENCE}

[1] P.Y. Chiou, Z. Chang, and M.C. Wu, "A Novel Optoelectronic Tweezer Using Light Induced Dielectrophoresis," Proceeding of IEEE/LEOS International Conf. Optical MEMS, pp. 8 9, 2003.

[2] A. Ashkin, J.M. Dziedzic, J.E. Bjorkholm, and S. Chu, "Observation of a single-beam gradient force optical trap for dielectric particles," Opt. Lett., Vol. 11, pp. 288-290, 1986.

[3] A. Ashkin and J.M. Dziedzic, "Optical trapping and manipulation of viruses and bacteria," Science, vol. 235, p. $1517,1987$.

[4] A. Ashkin, J.M. Dziedzic, and T. Yamane, "Optical trapping and manipulation of single cells using infrared laser beams," Nature, Vol. 330, p. 769, 1987.

[5] M. Ozkan, S. Bhatia, S.C. Esener, "Optical addressing of polymer beads in microdevices," Sensors and Materials, Vol. 14, No. 4,pp. 189-197, 2002.

[6] H.A. Pohl, Dielectrophoresis, Cambridge University Press, 1978.

[7] R. Schwarz, F. Wang, and M. Reissner, "Fermi-level dependence of the ambipolar diffusion length in amorphous silicon thin film transistors," Appl. Phys. Lett., Vol. 63, p. 1083, 1993. 